\section{Surgical checklist for cataract surgery: progress with the initiative by the Royal College of Ophthalmologists to improve patient safety}

SP Kelly' ${ }^{1}$ LR Steeples ${ }^{1}$, R Smith ${ }^{2}$ and A Azuara-Blanco ${ }^{3}$
'Department of Ophthalmology, Royal Bolton Hospital NHS Foundation Trust, Bolton, UK

${ }^{2}$ Department of Ophthalmology, Stoke Mandeville Hospital, Aylesbury, UK

${ }^{3}$ Centre for Vision and Vascular Science, Queen's University Belfast, Belfast, UK

Correspondence: SP Kelly, Department of Ophthalmology, Royal Bolton Hospital NHS Foundation Trust, Bolton BL4 OJR, UK.

Tel: + 44 (0)1204 390694;

Fax: $+44(0) 1204390442$.

Received: 25 June 2012 Accepted in revised form: 28 April 2013

Published online: 24 May 2013

Presented, in part, at the 2012 and 2013 Annual Congress of the Royal College of Ophthalmologists

\begin{abstract}
Purpose The World Health Organisation (WHO) identified patient safety in surgery as an important public health matter and advised the adoption of a universal perioperative surgical checklist. An adapted version of the WHO checklist has been mandatory in the National Health Service since 2010. Wrong intraocular lens (IOL) implantation is a particular safety concern in ophthalmology. The Royal College of Ophthalmologists launched a bespoke checklist for cataract surgery in 2010 to reduce the likelihood of preventable errors. We sought to ascertain the use of checklists in cataract surgery in 2012.

Patients and methods A survey of members of the Royal College of Ophthalmologists seeking views on the use of checklists in cataract surgery. Four hundred and sixty-nine completed responses were received (18\% response rate).

Results Respondents worked in England $(75 \%)$, Scotland $(\mathbf{1 1 \%})$, Wales $(5 \%)$, Northern Ireland (2\%), the Republic of Ireland (1\%), and overseas $(6 \%)$. Ninety-four per cent of respondents support the use of a checklist for cataract surgery and $85 \%$ say that they always use a checklist before cataract surgery. Sixtyseven per cent of cataract surgeons stated they undertake a pre-operative team brief. Thirty-six per cent use a cataract surgery checklist developed locally, $18 \%$ use the college's bespoke cataract surgery checklist, $39 \%$ use a generic surgical checklist, and $4 \%$ reported that they do not use a checklist.
\end{abstract}

Conclusion Ninety-three per cent of cataract surgeons responding to the questionnaire report using a surgical checklist and $67 \%$ use a team brief. However, only $54 \%$ use a checklist, which addresses the selection of the correct intraocular implant. We recommend wider adoption of checklists, which address risks relevant to cataract surgery, in particular the possibility of selection of an incorrect IOL.

Eye (2013) 27, 878-882; doi:10.1038/eye.2013.101; published online 24 May 2013

Keywords: surgical checklist; cataract; wrong implant; patient safety; human factors; non-technical skills

\section{Introduction}

In recent years there have been significant initiatives to improve patient safety in surgical care and specifically in cataract surgery. In 2008, the World Health Organisation (WHO) launched the 'Safe Surgery Saves Lives' initiative to improve surgical practice globally. ${ }^{1}$ The WHO scheme includes the use of a generic peri-operative checklist that has resulted in reductions in surgical mortality and morbidity. ${ }^{2}$ The WHO checklist was adapted and launched by the National Patient Safety Agency (NPSA) in 2009 for use within the National Health Service (NHS) in England and Wales. From 2010, the NPSA checklist, with the possibility for local modifications, became a mandatory requirement for all operations in NHS organisations in England. ${ }^{3}$ 
The Royal College of Ophthalmologists ('the College'), alongside the NPSA, launched a bespoke checklist in May 2010 for cataract surgery. ${ }^{4}$ The checklist covers safety factors and clinical risks pertinent to cataract surgery, such as intended refractive outcome and the lens implant type, and power predicted to achieve this outcome, which replace questions from the generic WHO checklist that are not relevant to cataract surgery (such as estimated blood loss). The College's cataract checklist aims to minimise the risk of avoidable errors, such as the selection of an incorrect intraocular lens (IOL). Implantation of an incorrect IOL leading to unplanned refractive error is one of the most frequent causes of litigation in ophthalmic care, ${ }^{5-7}$ and is classified by the NHS as a 'never event'.

We aimed to investigate the extent to which ophthalmologists in the United Kingdom have adopted the use of cataract surgery checklists.

\section{Materials and methods}

An invitation to participate in an online survey, using Zoomerang software (SurveyMonkey, Palo Alto, CA, USA), was sent electronically to all RCOphth members for whom email contact details were available. Only members who perform cataract surgery were asked to complete the survey. Data were collected prospectively over a 3-week period (March-April 2012). College members were asked to respond anonymously to questions on surgical checklist and 'team-brief' use before cataract surgery. The questions included grade of the responder, the location of base hospital, and the type of checklist used. Surgeons were asked of their opinion on the value of a checklist, including whether they considered a checklist to be 'too time consuming'. Those not using a checklist were asked to detail their reasons for not doing so. Free text comments were also invited. The data obtained were entered into a customised spreadsheet and were analysed using Microsoft Excel 2007.

\section{Results}

An invitation was sent electronically to 2856 College members. One hundred emails failed to deliver. In total, 496 completed responses were received. Sixty per cent ( $n=296)$ of responders were consultant ophthalmologists, representing a $24 \%$ response from consultants. Twentyseven per cent $(n=133)$ were trainees or fellows and $13 \%$ were non-consultant career grade doctors $(n=65)$. The overall response rate was $18 \%$. The majority of respondents $(93 \%)$ practice in the United Kingdom (England (75\%), Scotland (11\%), Wales (5\%), or Northern Ireland (2\%)). The remainder indicated they are based overseas $(6 \%)$ or in the Republic of Ireland (1\%).

\section{Checklist and team-brief use}

In total, $85 \%$ indicated they 'always' use a surgical checklist, $7 \%$ reported they 'often' used a checklist, and $4 \%$ said they 'sometimes' used a checklist. The remaining $4 \%(n=20)$ stated they 'never' use a checklist. The majority of surgeons (67\%) stated they 'always' use a 'team brief' before undertaking cataract surgery. Of the remainder $16 \%, 10 \%$, and $7 \%$ reported they 'often', 'sometimes', and 'never', respectively, use a 'team brief'.

Among consultant responders, 89\% indicated they always use a checklist and $71 \%$ indicated they always undertake a team brief before surgery. Among those $(n=20)$ indicating they do not use a checklist, the leading reasons stated were the presence of an established routine before surgery, non-availability of a checklist, and existing pre-operative nursing checks.

\section{Type of checklist used}

Four hundred and eighty-three surgeons reported the type of peri-operative checklist used for cataract surgery. Thirty-nine per cent $(n=186)$ reported using a generic surgical checklist proposed in their base hospital, $36 \%(n=174)$ use a cataract surgery checklist developed in their own hospital, $18 \%(n=89)$ use the College's bespoke cataract surgery checklist, $2 \%(n=10)$ reported using the WHO surgical checklist, and less than $1 \%(n=3)$ indicated 'other'. Figure 1 indicates the type of checklist used across the UK nations.

\section{Is a checklist considered of value?}

Ninety-four per cent $(453 / 481)$ of respondents stated that they consider that a pre-operative checklist to be of value in cataract surgery. Among those who stated they did not consider it of value, $60 \%$ still reported that they always use a checklist. Eighty-two per cent (392/478) indicated that they do not consider a checklist to be too time consuming.

\section{Free text comments}

Respondents suggested that data and questions included in the checklist should be relevant to cataract surgery with emphasis on intended refractive outcome, including IOL power, IOL availability, and planned incision site. A number of surgeons suggested the development of different forms for local and general anaesthetic cataract surgery to remove questions relevant to general anaesthesia before local anaesthetic cases (which represent the overwhelming majority of cataract procedures). Blood glucose level for patients with diabetes was highlighted as missing from the College 


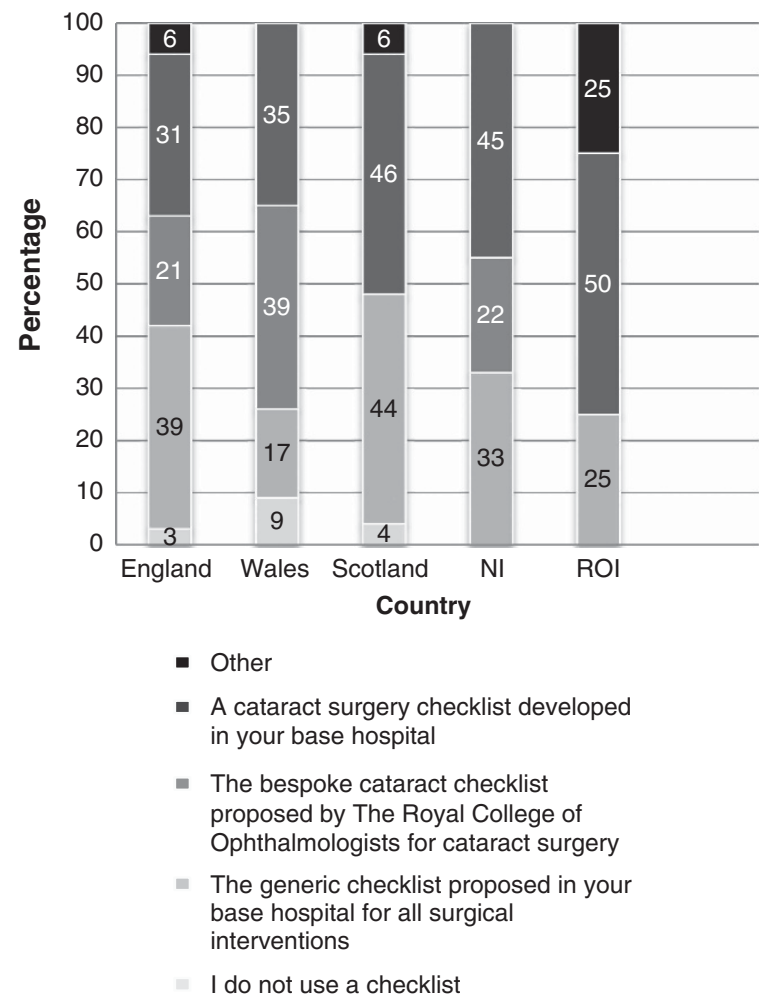

Figure 1 Type of checklist used per nation.

Table 1 Free text comments regarding the use surgical checklists for cataract surgery

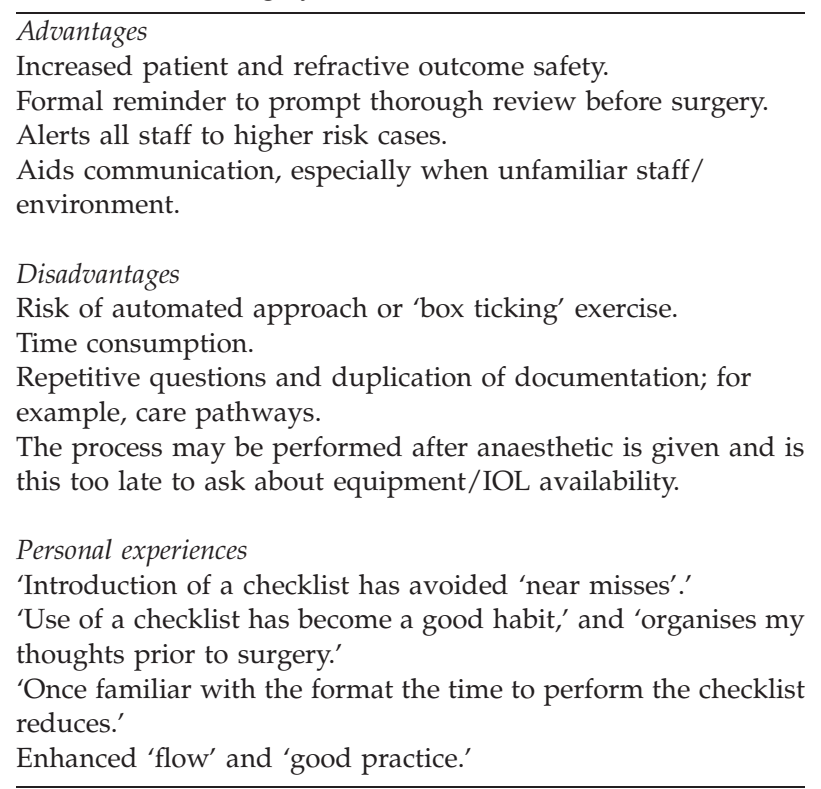

cataract checklist. Regarding generic checklists, a significant number of respondents highlighted that the questions are not always 'relevant' or 'appropriate' to cataract surgery, and feel that such questions diminish the credibility of the checklist. Table 1 presents key comments regarding advantages, disadvantages, and personal experiences of checklists in cataract surgery.

\section{Discussion}

Cataract removal with IOL implantation is the most common elective surgical procedure performed in the NHS ( 330000 surgeries per year) and in many other countries. Although serious problems with cataract surgery are rare, preventable errors can lead to poor outcomes and poor patient experience. This survey was undertaken to ascertain the adoption of peri-operative checklists in cataract surgery, since the introduction of the WHO generic checklists and the subsequent introduction of specific checklists for cataract surgery. It is encouraging to find that $85 \%$ of respondents 'always' use a checklist before cataract surgery, that $94 \%$ consider a checklist to be beneficial in cataract surgery, and that most users reported positive personal experiences of using a checklist.

Importantly, over half of cataract surgeons reported using a checklist designed specifically for cataract surgery. More surgeons reported using a locally designed cataract checklist (36\%) rather than the College's checklist (18\%). Similarly, amongst surgeons using a generic checklist, more use a locally developed version rather than the WHO checklist (2\%). The NPSA wisely encouraged adaptation of its generic version of the WHO Safer Surgery checklist for local or specialty use, with the proviso that local governance measures are followed, as it recognised that different procedures pose different risks to patient safety. The College cataract checklist was provided in an editable format to facilitate local modification. A number of free text comments indicated that some units mandate the use of a generic checklist and do not permit its adaptation for specialty procedures, possibly reflecting a misguided belief that any deviation from the generic checklist was either not permitted or that adaptation would undermine the adoption of a checklist as standard practice.

Most (67\%) cataract surgeons stated they undertake a pre-operative team brief. A team brief at the start of a cataract operating list helps to ensure that the right equipment and environment will be in place and that any foreseeable difficulties are anticipated. The use of preoperative team briefings and checklists to promote better team communication have been shown to improve surgical care. ${ }^{8}$ The cataract patient checklist includes a 'time-out', which provides a vital final opportunity to check the patient, site, procedure, and the IOL required against source biometry documents before each operation. We provided a training film on such matters at the recent College Congress and is available online. ${ }^{9}$ 
A strength of our survey is that $60 \%$ of respondents were consultant ophthalmologists and represent nearly one quarter of the College's consultant membership. The survey is representative of the UK practice, as $93 \%$ of responders work within the United Kingdom. We acknowledge that not all College members and, specifically, not all of the 1227 consultants contacted undertake cataract surgery. We cautiously suggest that the 296 replies from consultants may represent a greater proportion of current consultants who undertake cataract surgery. Unfortunately, the College's membership database does not provide information to investigate further. We acknowledge the overall low response rate and that we did not survey non-surgical members of cataract teams.

It is recognised that a large proportion of adverse clinical events is due to organisational or human behavioural factors. Although there is no evidence to date that use of a checklist has reduced the incidence of adverse events in cataract care, the use of a checklist has been associated with reduction of morbidity and mortality in other surgical areas. ${ }^{2}$ Non-technical skills, such as decision making, awareness of changing situations, communication, teamwork, and leadership, have also recently been recognised as key to improving patient safety. ${ }^{10}$ Such skills have attracted relatively little attention or research to date in ophthalmic surgery, but have been found of value in other areas, such as anaesthesia and general surgery. ${ }^{11}$ Several Colleges in the United Kingdom and abroad have introduced non-technical skills into their training programmes, as there is increasing evidence to support this initiative. ${ }^{12-14}$ In general surgery, poor teamwork increases risk of peri-operative death or major complication. ${ }^{15}$

The Department of Health stated in 2011 that insertion of a wrong implant (an implant other than the one intended and resulting in an unplanned outcome requiring further treatment) is a 'never' event. ${ }^{16}$ The College recommends IOL selection before surgery, during the assessment clinic, or pre-operative 'ward round'. ${ }^{17}$ The checklist provides an additional safety net to ensure correct IOL selection. The American Academy of Ophthalmology has provided an educational resource on reducing wrong site/wrong IOL surgery. ${ }^{18}$ Further information on patient safety is available in the College website. $^{19}$

Direct evaluation of the impact of a pre-operative checklist in cataract surgery is difficult, because significant avoidable adverse events occur infrequently. However, an audit of the impact of a checklist has shown encouraging results with improved staff communication in an NHS district general hospital ophthalmic department. $^{20}$
Improving patient safety in cataract surgery is a challenging and evolutionary process, which extends beyond the use of checklists and team briefs. Use of surgical checklists reflects an evolving cultural awareness of the importance of safety in healthcare and service industries. The WHO renewed its commitment to global patient safety issues in 2012 with the launch of the WHO 'Patient Safety Programme'. The Patient Safety Programme aims to support the framing of patient safety policies and promotion of good practices in safety and quality improvements. ${ }^{21}$ The successful implementation and use of checklists requires surgical teams to adopt a culture where the safety of the patient becomes everyone's responsibility. Vats et al ${ }^{22}$ emphasised the need for good training, leadership, clarification of roles in completing the checklist, and the need for adaptation of the generic checklist. The medical director of the NHS in England, Sir Bruce Keogh, identified that there is more work to be undertaken on improving the safety of surgery, such as reducing 'never events' and achieving cultural change in clinical staff. ${ }^{23}$

Some surgeons remain sceptical that surgical checklists and briefings improve patient safety, but in fact a powerful link has been found between briefing practices and attitudes towards patient safety. ${ }^{24}$ To overcome the criticism that checklists and team briefs are time consuming, repetitive exercises processes, which duplicate existing processes, it is very important that they are focused and relevant to the procedures being undertaken. Rigid imposition of a generic checklist and team brief on all surgical lists will achieve nominal compliance but may fail to secure real commitment, and may fail to address risks that are specific to particular procedures but are not covered by a generic checklist. We therefore recommend that the adaptation of peri-operative checklists where appropriate should be encouraged, within local governance processes.

\section{Conclusion}

It is encouraging that the majority of cataract surgeons who responded to the survey now use a surgical checklist and team brief. The commonest trend is to use a cataract-specific checklist, although generic checklists are often used. We recommend widespread adoption of cataract-specific checklists with local personalisation and adaptation. Checklist use is associated with reduced surgical morbidity and mortality, and positive impact on team working and non-technical skills, which are imperative to improving patient safety. We suggest further investigation of the impact of checklists in cataract surgery safety, particularly in relation to 'never events.' 


\section{Summary}

\section{What was known before}

- A surgical checklist is advised by the WHO and is of value. A bespoke checklist for cataract surgery has been made available by the Royal College of Ophthalmologists. Errors in cataract care are infrequent, but are frequently preventable

What this study adds

- Many ophthalmologists are now undertaking team briefings and checklist use. This is encouraging. However there is room for improvement in the use of cataract surgery-specific checklist use.

\section{Conflict of interest}

SPK and SR declare they were involved, unpaid, in drafting the College's cataract surgery checklist. All other authors declare no conflict of interest.

\section{Acknowledgements}

We thank the College members who responded to this survey. We thank Miss Beth Barnes of the College for her help with the survey.

\section{References}

1 World Health Organisation. Surgical Safety Checklist 2009, Available at www.who.int/patientsafety/safesurgery/en/ index.html.

2 Haynes AB, Weiser TG, Berry WR, Lipsitz SR, Breizat AH, Dellinger EP et al. A surgical safety checklist to reduce morbidity and mortality in a global population. $N$ Engl J Med 2009; 360: 491-499.

3 National Patient Safety Agency. National Patient Safety Agency, Alert 0861. National Patient Safety Agency: London, 2009. Available at http://www.nrls.npsa.nhs.uk/alerts/ ?entryid $45=59860$

4 National Patient Safety Agency. WHO Surgical Safety Checklist: For Cataract Surgery Only. National Patient Safety Agency: London, 2010. Available at http:// www.nrls.npsa.nhs.uk/resources/clinical-specialty/ surgery $/$ ?entryid $45=74132$.

5 Kelly SP, Jalil A. Wrong intraocular lens implant; learning from reported patient safety incidents. Eye 2011; 25: 730-734.

6 Ali N, Little BC. Causes of cataract surgery malpractice claims in England 1995-2008. Br J Ophthalmol 2011; 95: 490-492.

7 Simon JW, Ngo Y, Khan S, Strogatz D. Surgical confusions in ophthalmology. Arch Ophthalmol 2007; 125: 1515-1522.

8 Lingard L, Regehr G, Orser B, Reznick R, Baker GR Doran $\mathrm{D}$ et al. Evaluation of a preoperative checklist and team briefing among surgeons, nurses, and anesthesiologists to reduce failures in communication. Arch Surg 2008; 143: 12-17.

9 Mohammed BR, Vakros G, Ford R, Azuara-Blanco A. Non-Technical Skills For Eye Surgery DVD Presented at the Royal College of Ophthalmologists Annual Congress 2012. Available at http://www.rcophth.ac.uk/page.asp?section= 442\&sectionTitle=Patient+Safety+Information.

10 Youngson GG, Flinn R. Patient safety in surgery: non-technical aspects of safe surgical performance. Patient Saf Surg 2010; 4: 4.

11 Azuara-Blanco A, Reddy A, Wilkinson G, Flin R. Safe surgery non-technical aspects. Eye 2011; 25: 1109-1111.

12 Dickinson I, Watters D, Graham I, Montgomery P, Collins J. Guide to the assessment of competence and performance in practising surgeons. ANZ J Surg 2009; 79: 198-204.

13 Flin R, Patey R, Glavin R, Maran N. Anaesthetists' non-technical skills (ANTS). Br J Anaesth 2010; 105: 38-44.

14 Flin R, Yule S, Paterson-Brown S, Maran N, Rowley D, Youngson $\mathrm{G}$. Teaching surgeons about non-technical skills. Surgeon 2007; 5: 86-89.

15 Mazzocco K, Petitti DB, Fong KT, Bonacum D, Brookey J, Graham $S$ et al. Surgical team behaviours and patient outcomes. Am J Surg 2009; 197: 678-685.

16 Department of Health. The Never Events List 2011/12. Department of Health: London, 2011. Available at http:/ / www.dh.gov.uk/prod_consum_dh/groups/ dh_digitalassets/documents/digitalasset/dh_124580.pdf.

17 The Royal College of Ophthalmologists. Cataract Surgery Guidelines 2010. The Royal College of Ophthalmologists: London, 2010. Available at http://www.rcophth.ac.uk/ core/core_picker/download.asp?id=544.

18 American Academy of Ophthalmology. Wrong Site/Wrong IOL Performance Improvement. http:/ /one.aao.org/ce/ practiceguidelines $/$ patient_content.aspx?cid $=\mathrm{d} 0 \mathrm{db} 838 \mathrm{c}-$ 2847-4535-baca-aebab3011217.

19 The Royal College of Ophthalmologists. Patient Safety Webpage. The Royal College of Ophthalmologists: London. Available at http://www.rcophth.ac.uk/page.asp?section= 442\&sectionTitle $=$ Patient + Safety+Information .

20 Reeves GMB, Salibi A, Kelly SP, Rowlands A. Do surgical checklists improve communication in ophthalmic operating theatres? A quality improvement report Poster Presented at the Royal College of Ophthalmologists Annual Congress 2011.

21 World Health Organisation. Patient Safety Programme. Available at http://www.who.int/patientsafety/about/ programmes/en/index.html.

22 Vats A, Vincent CA, Nagpal K, Davies RW, Darzi A, Moorthy K. Practical challenges of introducing WHO surgical checklist: UK pilot experience. BMJ 2010; 340: 5433.

23 Safer surgery - the continuing challenge. Available at http://www.patientsafetyfirst.nhs.uk/Content. aspx?path=/Campaign-support/safer-surgery-week-2012/.

24 Allard J, Bleakley A, Hobbs A, Coombes 1. Pre-surgery briefings and safety climate in the operating theatre. $B M J$ Qual Saf 2011; 20: 711-717. 